\title{
Optimal Sensor Placement for Leakage Detection and Isolation in Water Distribution Networks *
}

\author{
Albert Rosich* Ramon Sarrate ${ }^{* *}$ Fatiha Nejjari ${ }^{* *}$ \\ * Institute of Robotics and Industrial Informatics, Edifici U, Pau \\ Gargallo, 5, 08028-Barcelona, Spain \\ ** Automatic Control Department, Universitat Politècnica de \\ Catalunya (UPC), Rambla de Sant Nebridi, 11, 08222 Terrassa, \\ Spain.
}

\begin{abstract}
In this paper, the problem of leakage detection and isolation in water distribution networks is addressed applying an optimal sensor placement methodology. The chosen technique is based on structural models and thus it is suitable to handle non-linear and large scale systems. A drawback of this technique arises when costs are assigned uniformly. A main contribution of this paper is the proposal of an iterative methodology that focuses on identifying essential sensors which ultimately leads to an improvement of the optimal search efficiency. The algorithm presented in this work is successfully applied to a District Metered Area (DMA) in the Barcelona water distribution network.
\end{abstract}

\section{Keywords: Optimal Sensor Placement, Leakage Detection, Water Distribution Networks}

\section{INTRODUCTION}

An important matter concerning water distribution networks is system water loss, which has a meaningful effect on both water resource savings and costs of operation (Farley, 2003). Continuous improvements on water loss management are being applied. New technologies are developed to achieve higher levels of efficiency, intended to reduce losses to acceptable levels considering technical and economical aspects. Usually a leakage detection method in a District Metered Area (DMA) starts analysing input flow data, such as minimum night flows and consumer metering data. Once the water distribution district is identified to have a leakage, techniques are used to locate the leakage for pipe replacement or repairing. The whole process could take weeks or months with an important volume of water wasted. To overcome this problem, different leakage detection and localisation techniques are carried out in the field. One of such techniques is the use of mathematical models (Brdys, 1994) which permits comparing the data gathered by installed sensors in the network with the data obtained by a model of this network. If a difference is detected between these data sets, a detection of an abnormal event is obtained. Thus, modelling is paramount in order to achieve successful results. This model is the mathematical tool linking the real sensor data gathered from the network to the decision making procedure. The tool provides fault detection as well as their probable location in the network.

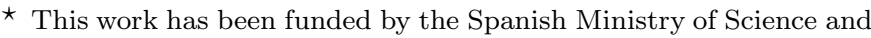
Technology through the CICYT project WATMAN (ref. DPI200913744), by the Spanish Ministry of Economy and Competitiveness through the CICYT project SHERECS (ref. DPI2011-26243), and by the European Commission through contract i-Sense (ref. FP7-ICT2009- 6-270428)
}

Fault diagnosis systems are an increasing and important topic in many industrial processes. The number of publications devoted to fault diagnosis has increased notably in the last years, as it can be seen in Blanke et al. (2006). In model-based fault diagnosis, diagnosis is basically performed from the responses of residual generators. Residual generators are functions obtained from the model which perform the task of comparing the process model and on-line process information. Since process information is usually obtained by means of the sensors installed in the process, it is important to develop methodologies to place the correct sensor set in the process in order to guarantee some diagnosis specifications.

Some results devoted to sensor placement for diagnosis can be found in Raghuraj et al. (1999), Krysander and Frisk (2008), Commault et al. (2008), Yassine et al. (2008), Travé-Massuyès et al. (2006), and Rosich et al. (2007). All these works use a graph-based approach and define different diagnosis specifications to solve the sensor placement problem. In particular, the present paper proposes the study of which pressure sensors need to be installed in order to improve the capability of detecting and isolating leaks in a DMA. The methodology presented in Rosich et al. (2010) will be applied to a real DMA network located in Barcelona.

The paper is organized as follows: In Section 2, the sensor placement problem tackled in this paper is formally introduced. Next, Section 3 presents the diagnosis framework based on structural models. Section 4 introduces the algorithms used to solve the aforementioned problem, whereas computation and implementation issues of these algorithms are discussed in Section 5. In Section 6, a 
real DMA network in Barcelona is analysed. Finally, some conclusions and remarks are given in Section 7.

\section{PROBLEM FORMULATION}

The aim of the sensor placement for fault diagnosis can be roughly stated as the choice of a sensor configuration such that a fault diagnosis performance specification is fulfilled. Usually, several sensor configurations that satisfy the required fault diagnosis specification will exist. Giving a cost to every sensor configuration makes it possible to define an optimal sensor placement problem, where the best sensor configuration is sought.

Let $\mathbf{S}$ be the set of candidate sensors. Any sensor $s \in \mathbf{S}$ can be chosen for installation, involving a cost denoted by $C(s)$. Such cost can comprise different concepts such as the purchase price, the maintenance price, the sensor reliability or the measurement precision, for instance. Given a sensor configuration $S \subseteq \mathbf{S}$, its cost will be defined as $\mathcal{C}(S) \triangleq \sum_{s \in S} C(s)$.

Let $\mathbf{F}$ be the set of faults that must be monitored. In a water distribution domain a leakage is an example of a fault, but other damages could be considered such as pipe blocking or tank overflow. The diagnosis specifications will be stated based on two properties: fault detectability and fault isolability. In this work, the single fault assumption will hold (i.e., multiple faults will not be covered) and no candidate sensor fault will be considered.

A fault $f \in \mathbf{F}$ is detectable if its occurrence can be monitored, whereas a fault $f_{i}$ is isolable from a fault $f_{j}$ if the occurrence of $f_{i}$ can be monitored independently of the occurrence of $f_{j}$. A formal definition of these two properties can be found in Krysander (2006). The fault detectability specification will be defined as:

$$
F_{\mathcal{D}}=\{f \in \mathbf{F} \mid f \text { is detectable }\}
$$

whereas the fault isolability specification will be defined as $\mathbb{F}_{I}=\left\{\ldots, F_{\mathcal{I}}\left(f_{i}\right), \ldots\right\} \quad \forall f_{i} \in F_{\mathcal{D}}$, with:

$$
F_{\mathcal{I}}\left(f_{i}\right)=\left\{f_{j} \in F_{\mathcal{D}} \mid f_{i} \text { is isolable from } f_{j}\right\} .
$$

To solve the sensor placement problem a system description $\mathcal{M}$ is required. Such description will allow the characterization of the fault diagnosis specifications based on the observations provided by the chosen sensors.

The optimal sensor placement for fault diagnosis is here formally stated as follows:

GIVEN A set of candidate sensors $\mathbf{S}$, a sensor cost function $C(\cdot)$, a system description $\mathcal{M}$, a fault detectability specification $F_{\mathcal{D}}$ and a fault isolability specification $\mathbb{F}_{I}$, FIND the minimum cost sensor configuration $S^{*} \subseteq \mathbf{S}$, such that the fault diagnosis specifications are fulfilled.

\section{STRUCTURAL FRAMEWORK FOR FAULT DIAGNOSIS}

A structural model will be used to solve the sensor placement problem. The analysis of the model structure has been widely used in the area of model-based diagnosis
(Blanke et al., 2006). The structural model of a system is an abstraction of the analytical model. In fact, the structural model is a coarse model simplification since only the relation between variables and equations is taken into account, neglecting the mathematical expression of this relation. Due to its simple description, it cannot be ensured that the diagnosis performance obtained from structural models will hold for the practical case. However if the required diagnosis performance is not fulfilled for a structural model, neither it will be for the practical case (i.e. only best case results can be computed).

A structural model is formalized as a bipartite graph $G(M, X, A)$, where $M$ is a set of model equations, $X$ a set of unknown variables and $A$ a set of edges, such that $\left(e_{i}, x_{j}\right) \in A$ as long as equation $e_{i} \in M$ depends on variable $x_{j} \in X$.

Structural modelling is suitable for an early stage of the system design, when the precise model expressions are not known yet, but it is possible to determine which variables are related to each equation. Furthermore, the diagnosis analysis based on structural models are performed by means of graph-based methods which have no numerical problems and are more efficient, in general, than analytical methods.

The Dulmage-Mendelsohn (DM) decomposition (Dulmage and Mendelsohn, 1958) is a well-known theoretical tool in the structural model-based fault diagnosis community. The DM decomposition defines a partition on the set of equations and the set of unknown variables of the structural model. Three main parts of $M$ can be identified in the partition, namely, the under-determined part, the just-determined part and the over-determined part. In the over-determined part, there are more equations than unknown variables, which implies that there exists some degree of redundancy, and this is the part of the model that is useful for monitoring the process. In the following, the notation $M^{+}$will represent the over-determined part of a given set of model equations $M$.

Next, the structural characterisation of the fault diagnosis properties of a system are briefly recalled (Krysander and Frisk, 2008). Without loss of generality, it is assumed that a single fault $f \in \mathbf{F}$ can only violate one equation, denoted by $e_{f} \in M$. In the case that a fault $f$ affected a subset of equations $E_{f} \subseteq M$, a new unknown variable $x_{f}$ and (fault) equation $e_{f}$ could be defined such that $x_{f}$ is related to $E_{f} \cup\left\{e_{f}\right\}$, which brings us back to the previous assumption.

Given a set of faults $F$, the following set is defined $M_{F}=$ $\left\{e_{f} \mid f \in F\right\}$.

Definition 1. A fault $f \in F$ is (structurally) detectable in a model described by the set of equations $M$ if

$$
e_{f} \in M^{+}
$$

Definition 2. A fault $f_{i}$ is (structurally) isolable from $f_{j}$ in a model described by the set of equations $M$ if

$$
e_{f_{i}} \in\left(M \backslash\left\{e_{f_{j}}\right\}\right)^{+}
$$

Example 1. Consider the equations set $M=\left\{e_{1}, e_{2}, e_{3}, e_{4}\right\}$ and the corresponding structural model, represented by the biadjacency matrix: 


$$
\begin{array}{ll}
e_{1}: & h_{1}\left(x_{1}, x_{2}, f_{1}\right)=y_{1} \\
e_{2}: & h_{2}\left(x_{1}, x_{2}, f_{2}\right)=y_{2} \\
e_{3}: & h_{3}\left(x_{1}\right)=y_{3} \\
e_{4}: & h_{4}\left(x_{2}\right)=y_{4}
\end{array}
$$

\begin{tabular}{c|cc} 
& $x_{1}$ & $x_{2}$ \\
\hline$e_{1}$ & $\times$ & $\times$ \\
$e_{2}$ & $\times$ & $\times$ \\
$e_{3}$ & $\times$ & \\
$e_{4}$ & & $\times$
\end{tabular}

where $x_{i}$ are unknown variables, $y_{i}$ are known variables and $h_{i}$ are nonlinear functions.

Assume two faults $f_{1}$ and $f_{2}$ that affect equations $e_{1}$ and $e_{2}$ respectively. Then, since $e_{1}, e_{2} \in\left\{e_{1}, e_{2}, e_{3}, e_{4}\right\}^{+}$, $e_{1} \in\left\{e_{1}, e_{3}, e_{4}\right\}^{+}$and $e_{2} \in\left\{e_{2}, e_{3}, e_{4}\right\}^{+}$, and according to Definitions 1 and 2 , both faults are detectable and isolable of each other.

These definitions will be next used in a structural approach for solving the sensor placement problem.

\section{OPTIMAL SENSOR PLACEMENT ALGORITHM}

In this section the approach proposed in Rosich et al. (2010) is recalled. The problem stated in Section 2 involves solving the following two issues: a search strategy for a candidate solution for the optimal sensor placement problem and the verification of the fault diagnosis specifications fulfilment of this candidate solution.

The first issue is solved by Algorithm 1. The search strategy is based on a depth-first search by choosing first the nodes with lowest costs and back-tracking to other not already explored nodes when a branch exploration is aborted.

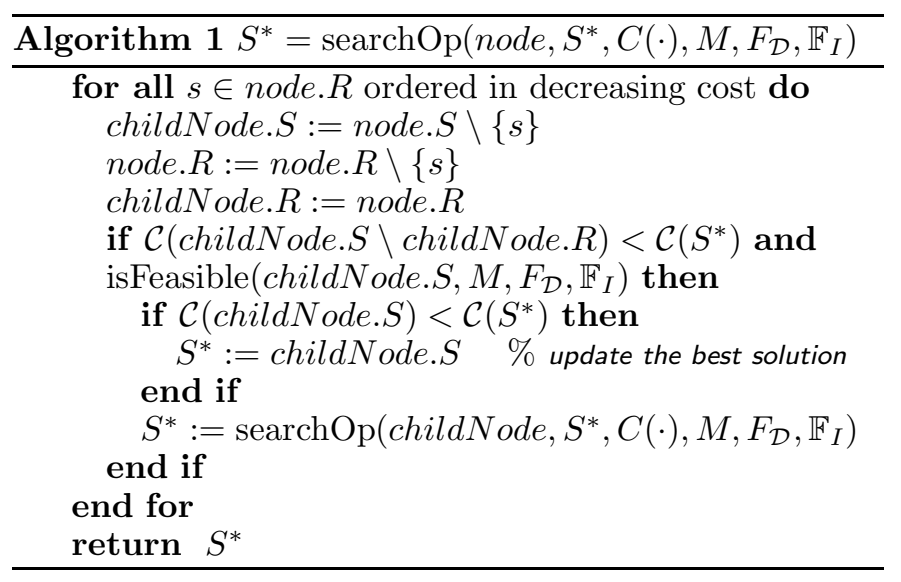

Every node in the tree consists of two sensor sets:

- node.S, the sensor configuration that the node represents (i.e., a candidate solution to test).

- node.R, the sensors that are allowed to be removed in its sub-nodes.

Throughout the search, the best solution is updated in $S^{*}$, whenever a feasible solution with a lower cost than the current best one is found. A branch exploration is aborted at some node when any of the following two conditions is fulfilled:

Condition 1: The lowest reachable cost by exploring sub-nodes of the current node is not lower than the cost of the current best solution.

Condition 2: The node is not a feasible solution for the sensor placement problem.
Remark that Condition 1 implies that there is no better sub-node in the branch than the current best solution. On the other hand, Condition 2 implies that no sub-node is neither a feasible solution.

Algorithm 1 is initialised according to the following criteria:

(a) The root node of the search tree corresponds to the candidate sensor set: node. $S:=$ node. $R:=\mathbf{S}$.

(b) The current best sensor configuration corresponds to the candidate sensor set: $S^{*}:=\mathbf{S}$.

(c) The cost corresponding to the candidate sensors.

(d) The set of equations corresponding to the fault-free behaviour of the system, $M$.

(e) Maximum fault diagnosis specifications will be sought. These are the specifications that are fulfilled when all candidate sensors are chosen. This is consistent with initialisation item (b).

The issue concerning the verification of the fault diagnosis specifications is addressed by Algorithm 2. Performing the sensor placement analysis involves the addition of new measurements to the system model. This implies that the original system model $M$ has to be updated accordingly.

Without loss of generality, it is assumed that a sensor $s_{i}$ can measure one single unknown variable $x_{i} \in X$. In the structural framework, such sensor will be represented by one single equation of the form $y=x_{i}$ (where $y$ is a measurement) and denoted as $e_{s}$. Given a set of sensors $S$, the following set is defined $M_{S}=\left\{e_{s} \mid s \in S\right\}$. Thus, given a candidate sensor configuration $S$ and a model $M$, the updated system model corresponds to $M \cup M_{S}$.

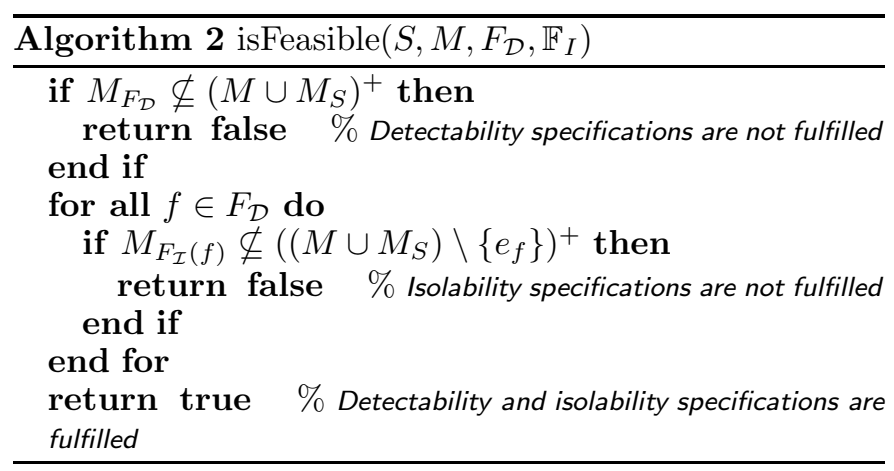

Remark that Algorithm 2 first checks the fault detectability specification according to Definition 1 for all $f \in F_{\mathcal{D}}$. Next, the fault isolability specification $\mathbb{F}_{I}$ is verified according to Definition 2 for all fault pairs $f_{i}, f_{j} \in F_{\mathcal{D}}$.

\section{OPTIMAL SEARCH EFFICIENCY ANALYSIS}

The computational complexity of Algorithm 2 depends on the DM decomposition operation, which is $m^{2.5}$ (Murota, 2000 ), with $m$ being the number of equations in the model. Thus, the algorithm computation time is not an issue.

Algorithm 1 is exponential in the number of candidate sensors. Given a set of $n$ candidate sensors, the worst case search occurs when the optimal sensor configuration includes $n / 2$ sensors, and involves visiting $2^{n} / 2$ nodes. In fact, the efficiency of the algorithm strongly depends on how the sensor costs are set. The more distinctly costs are assigned the better the algorithm performs. So, once 
a local lower cost solution is found, large branches of subnodes can be pruned from the search tree, leading to a more efficient search.

When the cost is assigned uniformly over the candidate sensors, special attention has to be paid regarding the order in which Algorithm 1 removes sensors from the set node. $R$. It is recommended to remove sensors ordered in decreasing essentiality. The more feasible sensor configurations a sensor appears in, the more essential the sensor is. Prioritizing the removal of essential sensors implies, according to Condition 2, that the exploration of the first branches terminates at the very initial nodes. Thus, a larger number of sub-nodes are dismissed during the search.

Therefore, in order to identify those essential sensors and to efficiently perform the optimization, the following iterative procedure is proposed:

(1) Define an arbitrary order on the node. $R$ sensor set corresponding to the root node.

(2) Launch Algorithm 1 and find the first locally optimal sensor configuration.

(3) Modify the order on the node. $R$ sensor set corresponding to the root node, by moving the sensors of this locally optimal configuration to the beginning. Remark that these sensors have some degree of essentiality since they are part of an intermediate solution.

(4) Repeat steps (2) and (3) until no more new sensors are found.

(5) Solve the globally optimal sensor placement problem by means of Algorithm 1, initialised with the last ordered node. $R$ sensor set obtained in the previous step.

A local optimum is found whenever Algorithm 1 performs back-tracking, i.e. a node of an upper level is generated in order to explore a new branch.

\section{APPLICATION TO A WATER DISTRIBUTION NETWORK}

This section shows how to solve the aforementioned problem for the case of a specific water distribution network. First, the DMA network is introduced where the most relevant features from the diagnosis perspective are given. Then, the structural model representation of this network is presented. And, finally the sensor placement problem is solved, obtaining encouraging results.

\subsection{Water network description}

The DMA network used as the case study in this work is located in the Barcelona area (see Figure 1). It has 881 nodes and 927 pipes. The network consists of 311 nodes with demand (RM type), 60 terminal nodes with no demand (EC type), 48 nodes hydrants without demand (HI type), 14 dummy valve nodes without demand (VT type) and 448 dummy nodes without demand (XX type). The network has two inflow inputs modeled as reservoir nodes.

Leakage detection is based on the premise that damage (leakage) in one or more locations of the piping network

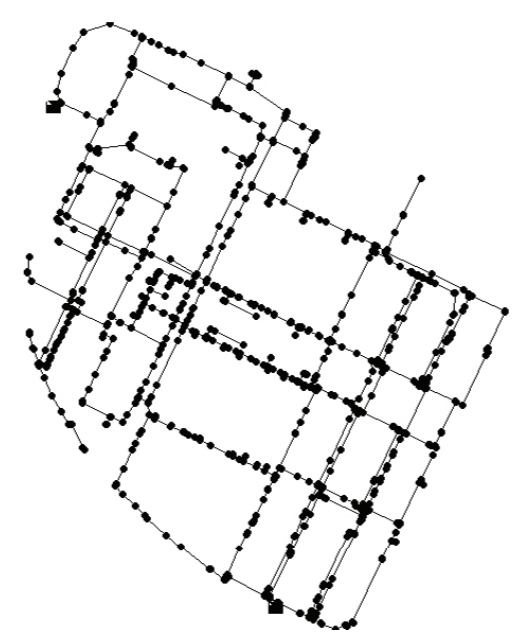

Fig. 1. Case study network map

involves local liquid outflow at the leakage location, which will change the flow characteristics (pressure heads, flow rates, acoustics signals, etc.) at the monitoring locations of the piping network.

Leaks might appear anywhere in the water network. However, due to modelling limitations, leaks are represented in the nodes where the flow balances take place. Therefore, a node with a leak can be modeled as

$$
\sum q_{\text {in }}-\sum q_{\text {out }}=q_{f}
$$

where $q_{\text {in }}$ are the input flows, $q_{\text {out }}$ are the output flows and $q_{f}$ is the outflow caused by the leak. In this case, $q_{f}$ is considered an unknown input of the system. Furthermore, the set of dummy nodes (XX type) represent the defects causing leaks in the network. Thus, there are 448 leaks to detect and isolate.

Hydraulic sensors may monitor pressure or flow rate. This work focuses on the placement of pressure monitoring points. They are used more frequently than flow rate sensors since it is cheaper and easier to collect pressure data, and the pressure transducers give instantaneous readings whereas most flow meters do not react instantaneously to flow variations (de Schaetzen et al., 2000). Flow rates are usually measured at all entry points to the network, on main pipes at the entrance into sub-networks, and/or at the outlet of elevated tanks and pumping stations. Thus, the selection of flow rate measurement points is straightforward and is limited to specific locations.

Therefore, only pressure sensors will be considered in the sensor placement problem. In order to reduce the problem complexity, just a subset of pressures is chosen as candidate variables to be measured. This subset consists of pressures in RM type nodes. There exist 311 pressures that can be measured in these nodes, so they represent the candidate sensor set. It is also assumed that there is no sensor previously installed in the network before solving the sensor placement problem.

\subsection{Structural model extraction}

As it was shown in Section 3, the diagnosis framework is based on structural models. Next, it will be explained how the corresponding structural model of the DMA 
water network is systematically obtained from the network graph.

The DMA network is originally represented as a direc graph $G=(N, L)$ where pipe junctions are nodes, $N$, c pipes are edges, $L$. Each node represents, at the sa time, a pressure variable and a flow balance equati Similarly, each edge represents a flow variable and a $\mathrm{p}$ equation. Therefore, given any node $n \in N$, the follow flow balance equation can be derived,

$$
\sum_{q_{i} \in Q_{n}} q_{i}=d_{n}
$$

where $Q_{n}$ represents all the flows of the edges incident node $n$, and $d_{n}$ is the known flow demand associated node $n$. Furthermore, given an edge $l \in L$, the correspo ing pipe equation can be deduced as

$$
q_{l}=\operatorname{sgn}\left(p_{i}-p_{j}\right) \cdot c\left(\left|p_{i}-p_{j}\right|\right)^{\gamma}
$$

where $q_{l}$ is the flow of edge $l, p_{i}$ and $p_{j}$ are the pressu of the nodes adjacent to edge $l=\left(n_{i}, n_{j}\right)$, and $c$ and $\gamma$ parameters modelling physical properties of the pipe, such as length, inside diameter, minor losses, and roughness.

Now, the structural model of the water network can be defined as the bipartite graph involving the equation node set $M$ and the unknown variable node set $X$. Let $M_{N}$ be the set of flow balance equations and $M_{L}$ be the set of pipe equations, so $M=M_{N} \cup M_{L}$. Note that there are as many equation in $M_{N}$ as nodes in $G$ and as many equations in $M_{L}$ as edges in $G$. Thus, this means 1810 equations for the Barcelona DMA network used here. On the other hand, let $Q$ be the set of flow variables and $P$ be the set of pressure variables, then it holds that $X=Q \cup P$. Therefore, the number of unknown variables is 1810 . The edges of the structural model are defined from the graph $G$, according to (6) and (7). In Figure 2, the resulting structural model is depicted in biadjacency matrix form where the equation set corresponds to rows and the variable set corresponds to columns. A dot in the $(i, j)$ element indicates that there exists an edge incident to equation $e_{i} \in M$ and variable $x_{j} \in X$, i.e., $\left(e_{i}, x_{j}\right) \in A$. Note that the structural model of the DMA network is a just-determined model where all unknown variables can be computed, i.e. the model could be used for simulation.

\subsection{Sensor placement for leak detection and isolation}

It is important to see that when a leak is present in a dummy node (XX type), the corresponding equation (6) does no longer hold. Indeed, a term $q_{f}$ should be added to the equation so that the model becomes consistent with the faulty water network. However, since detecting inconsistencies in the equation is the objective of modelbased diagnosis, the term $q_{f}$ is omitted and the set of faults, or leaks, is now represented as the subset of structural model equations in $M_{N}$ related to dummy nodes. Therefore, the following set of fault equations is defined

$$
M_{F}=\left\{e \in M_{N} \mid e \text { comes from an XX type node }\right\}
$$

The set of sensors is characterized by the subset of pressure variables in $P$ such that its corresponding node is a $\mathrm{RM}$ type node. When a sensor measuring pressure $p_{i}$ is placed, an equation in the form of $p_{i}=\hat{p}_{i}$ is added to the structural model, where $\hat{p}_{i}$ is the known measured value.

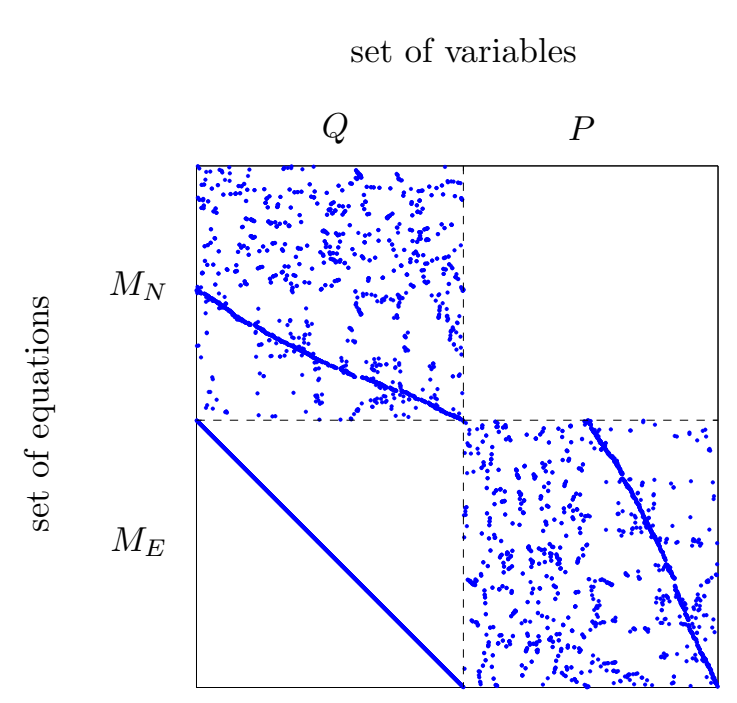

Fig. 2. Structural model of the DMA network

Before the sensor placement problem is solved, the maximum leak detection and isolation specifications must be determined. This can be straightforwardly done by placing all candidate sensors in the model and then performing diagnosability analysis according to Definitions 1 and 2 . The results obtained from this analysis are that all leaks can be detected, and 417 out of 448 leaks can be completely isolated from any other leak. Moreover, there are 16 leaks that are pairs-wise non-isolable (they can be isolated from any other leak except the paired one), two sets of three non-isolable leaks each, a set of 4 non-isolable leaks and a set of 5 non-isolable leaks. In conclusion, there are 31 out of 448 leaks that can not be completely isolated and in the worst case, when one of the 5 non-isolable leaks is present, we will not be able to isolate the correct one, among the 5 leaks.

The optimal sensor placement algorithm presented in Section 4 is now applied to the Barcelona DMA water network. Since all candidate sensors are of the same type (all of them measure pressure), the cost of installing a sensor is assumed to be equal for all candidate sensors. Therefore, solving the sensor placement problem involves finding the the minimum cardinality sensor set that satisfies the maximum leak detection and isolation specifications.

When applying Algorithm 1, sensors are removed from the set node. $R$ in arbitrary order. This leads Algorithm 1 not to find a solution in a reasonable time. In fact, Algorithm 1 run for three days without finding a solution, so the search was deliberately aborted. Thus, the methodology suggested in Section 5 is followed. The procedure involves up to 4 iterations and, in the end, 19 sensors are identified as essential. Next, in the last iteration a global optimum is searched. The optimal sensor configuration is then found in just 55.5 seconds. The 12 nodes corresponding to the optimal sensors are labeled in Figure 3. The optimal solution seems reasonable since it involves sensors related to nodes located in peripheral loop-free branches in the graph. This is mainly due to the fact that redundancy concerning nodes located in loop-free branches is more difficult to 


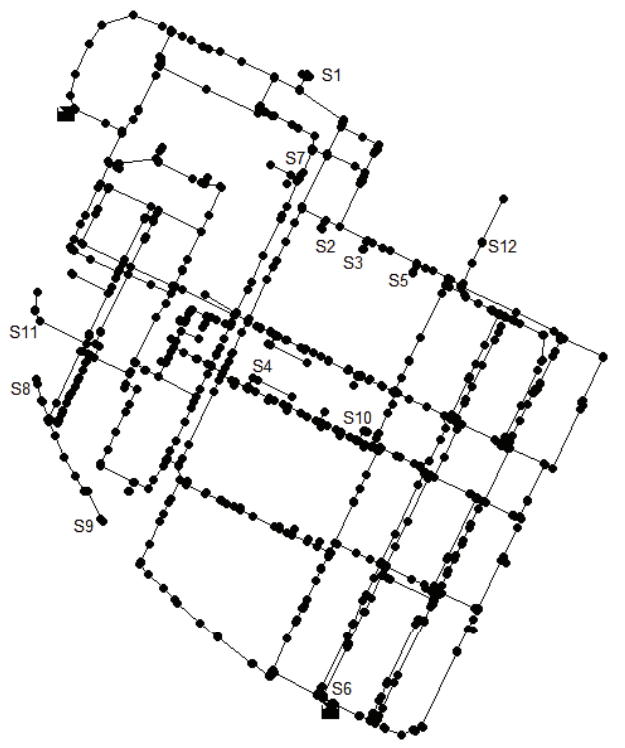

Fig. 3. DMA Optimal sensor location

attain than redundancy concerning nodes located in loops.

Algorithm 1 has been proved to be highly efficient. Given a set of 311 candidate sensors, the sensor placement algorithm searches a solution among $2^{311}$ potential sensor configurations. Obviously, checking all of them would be unaffordable. However, once the essential sensors have been identified, Algorithm 1 just needs to traverse 714392 sensor configurations. Of these, the fault diagnosis specifications are only verified against 4740 sensor configurations.

Note that the same diagnosis capabilities obtained by installing all 311 candidate sensors, are now achieved by just installing 12 sensors, which is a significant improvement regarding to the investment that the water company should confront.

\section{CONCLUSIONS}

The sensor placement problem in water distribution networks has been addressed in this paper. A water distribution network usually describes a mesh topology involving hundreds of nodes and pipes. Moreover, the behaviour of this kind of systems is governed by non-linear physical laws. Such complexity requires the development of tools applicable to non-linear large-scale systems. Therefore, a key contribution of this paper is the choice of a sensor placement technique that it is based on a structural model of the water distribution network.

Moreover, a second contribution of this paper is a procedure which improves the efficiency when applying Algorithm 1. This improvement aims at identifying essential sensors, which are prioritized when Algorithm 1 removes sensors with the same cost. Such improvement has been clearly demonstrated when applied to the water distribution network.

In this paper, a uniform cost has been assigned to every candidate sensor. However, Algorithm 1 is ready to be applied in case an heterogeneous cost distribution was assigned. For instance, even if just pressure transducers are considered, a different cost could be assigned to take into account node accessibility on a maintenance task.

The desired fault diagnosis specifications can be imposed to the search algorithm. This is an important feature, since the maximum fault diagnosis specifications are not always sought. Since the fault diagnosis performance is gained at the cost of sensor addition, if the budget for instrumentation was limited, the desired fault diagnosis specifications could be lightened accordingly.

\section{REFERENCES}

Blanke, M., Kinnaert, M., Lunze, J., and Staroswiecki, M. (2006). Diagnosis and Fault-Tolerant Control. Springer, 2nd edition.

Brdys, U. (1994). Operational control of water systems. Prentice Hall International, Prentice Hall International.

Commault, C., Dion, J.M., and Agha, S.Y. (2008). Structural analysis for the sensor location problem in fault detection and isolation. Automatica, 44(8), 2074-2080.

de Schaetzen, W.B.F., Walters, G.A., and Savic, D.A. (2000). Optimal sampling design for model calibration using shortest path, genetic and entropy algorithms. Urban Water, 2(2), 141-152.

Dulmage, A.L. and Mendelsohn, N.S. (1958). Covering of bi-partite graph. Canada J. Math, 10, 527-534.

Farley, T. (2003). Losses in Water Distribution Networks. IWA Publishing UK, UK.

Krysander, M. (2006). Design and Analysis of Diagnosis Systems Using Structural Analysis. Ph.D. thesis, Linköping Univ., Linköping, Sweden.

Krysander, M. and Frisk, E. (2008). Sensor placement for fault diagnosis. IEEE Trans. Syst., Man, Cybern. A, 38(6), 1398-1410.

Murota, K. (2000). Matrices and Matroids for Systems Analysis. Springer.

Raghuraj, R., Bhushan, M., and Rengaswamy, R. (1999). Locating sensors in complex chemical plants based on fault diagnostic observability criteria. AIChE J., 45(2), 310-322.

Rosich, A., Sarrate, R., Puig, V., and Escobet, T. (2007). Efficient optimal sensor placement for model-based FDI using and incremental algorithm. In Proc. 46th IEEE Conference on Decision and Control, 2590-2595. New Orleans, USA.

Rosich, A., Yassine, A.A., and Ploix, S. (2010). Efficient optimal sensor placement for structural model based diagnosis. In 21th International Workshop on Principles of Diagnosis (DX-10). Portland, USA.

Travé-Massuyès, L., Escobet, T., and Olive, X. (2006). Diagnosability analysis based on component supported analytical redundancy relations. IEEE Trans. Syst., Man, Cybern. A, 36(6), 1146-1160.

Yassine, A.A., Ploix, S., and Flaus, J.M. (2008). A method for sensor placement taking into account diagnosability criteria. Int. J. Appl. Math. Comput. Sci., 18(4), 497512 . 